\title{
PREFACE:
}

\section{Gender-Sensitive Research at the Max Planck Society}

Ulla Weber

Central Gender Equality Officer, Max Planck Society

Some people might assume that gender research, or gender-sensitive research, and basic research do not go together. A look inside the Max Planck Society over the last five to six years provides a different picture and reveals a close connection between both fields. One will find three levels in which gender research leads to crucial findings which can be used to optimize the framework conditions for research, the quality of research, and the impact it has on improving our society.

\section{GENDER RESEARCH: BASIS FOR A SMART GENDER EQUALITY STRATEGY}

Although it is well known, and almost everyone wants to do something about it, the scientific community still displays gender inequality in terms of personnel, which works to the detriment of female scientists and leads to a significant gender imbalance. ${ }^{1}$ Research organizations, including the Max Planck Society, have been addressing female under-representation in the sciences since the early 1990 s with a wide range of gender equality measures. Initially, these were mainly affirmative action measures for women, such as women-only positions, mentoring, training, and coaching -measures that were specifically aimed at "fixing" the women. All these

1 The structural discrimination of women in the labor market is not limited to science. The quantitative findings are numerous and sobering. Despite the continuous increase in the proportion of women in science as a whole, this figure drops dramatically the higher one climbs the career ladder, and the over-representation of male scientists in universities and research organizations is considerable. The A level professors and directors in German research organizations are 84 percent male, in universities 80 percent male. These figures are reflected in the number of publications and authorships, in the gender distribution among Nobel laureates, and even on Wikipedia women scientists are significantly less represented than their male colleagues. 
tools and programs were based on the assumption that females are somehow unfit for a scientific profession-unrelated to scientific knowledge, but focusing on strategic competencies. Our research organization implemented a leadership program, a fund to finance scientific positions for females only, a mentoring network, and various soft skill seminars to empower female scientists. Then, some years ago, the Max Planck Society changed their gender equality strategy, based on research findings.

Three observations led to this. First, there was the change of societal thinking at that time. Since then, various scientific studies have demonstrated the influence of gender stereotypes on performance evaluation and personnel selection in science. Second, it became obvious that although measures designed to support individual women achieved some good results in terms of the individual development of these women, and increased the number of female scientists in the short term, they do not change circumstances and achieve gender balance in the long term. Third, the Max Planck Society had to recognize that affirmative action was no longer on the cards-neither for female scientists, nor for the institutes. There was a decrease in demand for programs that were exclusively available to female scientists.

Max Planck Management therefore commissioned external researchers to conduct two studies. The aim of the first study was to internationally benchmark the portfolio of Max Planck gender equality measures. Furthermore, it explored the question of what tools the "big players" in international research had already implemented. ${ }^{2}$ The second project investigated the gender equality status quo at Max Planck by means of a survey that was conducted among junior researchers, the results of which indicated a greater need for action to achieve equal opportunities between men and women. ${ }^{3}$

Though starting from two completely different approaches, the results of both studies suggested the same-namely, a change of course for the

2 Dethloff, Nina (2015). Chancengleichheit in der Max-PIanck-Gesellschaft. Vergleich mit ausgewählten internationalen Forschungseinrichtungen. Internal report commissioned by the Max Planck Society.

3 Fraunhofer Gesellschaft (Martina Schraudner et al.) 2014. Chancengleichheit und Nachwuchsförderung in der Max-Planck-Gesellschaft. Ergebnisse quantitativer und qualitativer Befragungen unter Nachwuchswissenschaftlerinnen und Nachwuchswissenschaftlern. More than one thousand Max Planck junior scientists participated in an online survey. The investigation was supplemented by interviews with a number of Max Planck directors and scientists who had left the Max Planck Society. 
Max Planck gender equality strategy whereby the Max Planck Society should focus less on the individual, and more on the cultural characteristics of an organization. The findings revealed that the scientific community still displays gender bias when it comes to recruitment processes and performance evaluation, which disadvantage female scientists. Once more, the findings draw our attention to the fact that a sustainable gender balance at all scientific career levels will not be achieved as long as society and decision makers consider women less fit for science than men.

In order to overcome this issue, both researchers recommended not only supporting female scientists through financial and training measures, but also by using additional approaches to increase the objectivity of important career procedures. One proposed solution was the roll-out of comprehensive gender awareness training for decision makers and co-workers. A second promising instrument was the use of "behavior design". Instead of de-biasing people's attitudes, this instrument aims to decouple personal attitudes from organizational structures and procedures. ${ }^{4}$

Specialist knowledge is vital for the successful implementation of all these approaches. Which stereotypes are the ones that hinder the career development of women scientists? How do they ingrain themselves into the structures and procedures of the scientific community? Which measures are suitable for overcoming blind spots and discrimination? Which measures are actually counterproductive and tend to produce and reinforce stereotypes? What standards must procedures and processes meet to be immune to such distortions? The more we know about the influence of stereotyping in science, the faster we can know how to dispel traditional myths that exclude certain people and groups. To obtain such data, the Max Planck Society has been involved in various research projects. These include "Neue Wissenschaftskarrieren" and "FlexiShip" at the national level, and "GENERA" and "LeTSGEPs" at the European level. ${ }^{5}$

4 Bohnet, 2016.

5 Neue Wissenschaftskarrieren BMBF-Projekt (Programm Frauen an die Spitze) 2013-2015, https://www.gender-diversity.tu-berlin.de/gdo/projekte/abgeschlossene_ projekte/wissenschaftskarrieren; Flexship 2015-2016, Projekt der EAF Berlin, https: //www.eaf-berlin.de/projekt/flexibel-arbeiten-fuehrung/; GENERA - Gender Equality Network in the European Research Area 2015-2018 funded by the European Commission (Horizon 2020), genera-project.com; LeTSGEPs - Leading Towards Sustainable Gender Equality Plans in research performing organizationsfunded by the European Commission (Horizon 2020) 2020-2024, https://letsgeps.e $\mathrm{u} /$ 
Based on such benchmarks, and by comparing our own strategy with those of other national and international scientific institutions, the research society has been able to reflect on its own status and employ tailormade measures and programs that are customized to Max Planck's professional and organizational culture. A perfect match is crucial for the success of the tools being used. However, research can do far more than just provide evidence-based measures to improve the science system.

\section{IMPROVING RESEARCH BY INTEGRATING GENDER ASPECTS}

Within engineering and applied science, one can find numerous examples of the need to integrate a gender dimension into different research designs. For example, research on the risks of osteoporosis in men has long been neglected. It is now known that about one third of men are affected and require effective therapies. Another breakthrough was the recognition that crash test dummies, designed according to male body models, do not adequately represent injury risks for females. On the other hand, a few years ago, research was able to show that the essential factor for an appropriate model of a knee implant has less to do with gender, as was long assumed, and more to do with the characteristics of height, ethnicity, and body composition. Impressive examples of the complexity of integrating gender aspects into technical and scientific developments can also be found at international and intercultural level. In these settings, it becomes clear how gender roles and gender-specific attributions may change the link with other culture-set categories. For example, the sustainable improvement of water infrastructure in some African countries can only be achieved by taking into account the role of the women who are responsible for fetching water there. ${ }^{6}$

As we have learned, integrating a gender dimension into applied science has improved, and will continue to improve, the well-being of societies world-wide enormously. Of course, the quality of applied science depends on the quality of basic research, because the former benefits from the findings of the latter. Conversely, almost every finding in basic research has implications for applied science. Therefore, it is crucial to understand the

6 More detailed information about these findings, and more examples for the successful integration of the gender dimension into science, can be found at the Stanford Website "Gendered Innovations in Science, Health \& Medicine, Environment, and Engineering” at http://genderedinnovations.stanford.edu/. Last accessed: June 18, 2020. 
impact of gender aspects on all research fields. Basic research can help uncover the "gender data gap" 7 which has to be closed in order to develop gender-equitable technology, medical treatment procedures and environmental solutions. Needless to say, the quality of scientific findings increases when a multitude of perspectives and approaches are taken into account.

With reference to this insight, the German Research Society (DFG) recently embedded gender and diversity dimensions into its "Guidelines for Ensuring Good Scientific Practice". They added a section on the relevance of gender and diversity to the funding application guidelines. In the future, applicants need to indicate whether, and to what extent, gender or other personal and social characteristics are relevant to methods, work programs and objectives of a research project. This information is required with respect to the scientists involved in the project, project participants, any persons affected by the research results and all other aspects.

\section{FUNDAMENTAL QUESTIONS. GENDER DIMENSIONS IN MAX PLANCK RESEARCH PROJECTS}

This book presents a selection of gender-sensitive research at the Max Planck Society-a selection that reflects the diversity of the Max Planck Institutes. The spectrum of contributions compiled for this anthology is broad. It contains a wide variety of approaches, topics, issues and methodological procedures. Some chapters deal with gender-specific research issues and some integrate gender equality aspects into certain research questions. Others examine the influence of gender aspects on research questions and results (Gendered Innovations). There are chapters which discuss gender participation in science, and chapters that reflect a gender-sensitive and gender-political research perspective. Not only do these chapters cover a broad range of topics, they also come from a wide variety of research fields. Most of the contributions come from the humanities, including law, art history, and the history of science. However, there is also an article on neuroscience and another on computer science.

Isabel Valera's contribution (Max Planck Institute for Intelligent Systems) deals with the highly charged topic of discrimination in algorithmic decision-making. Given that automated and data-driven decision-making processes are becoming more and more prevalent (spam filtering, product 
personalization, pre-trial risk assessment, mortgage approvals, etc.), the researchers around Isabel Valera are asking for tools and methods to reduce discrimination towards people from certain demographic groups (e.g., gender) and the impact of stereotypes within algorithmic decision-making. They are developing new definitions of what "fairness" means and are helping with the development of new algorithmic solutions by developing standards for the design of fair and differentiating classifiers. Julia Sacher and Rachel Zsido (Max Planck Institute for Human Cognitive and Brain Sciences) highlight two areas where gender has not been taken into account in neuroscience and present a strong case for this to be addressed immediately. Many basic and clinical neuroscience studies do not include equal numbers of females in their samples, and even fewer present a sexspecific analysis of their data. The strategies presented by Sacher and Zsido open up promising new perspectives on the diversity of human cognition and brain plasticity.

The Humans Sciences Section of the Max Planck Society is the source of a very different topic. Lisa Hanstein (Kunsthistorisches Institut in Florenz - Max Planck Institute) examines the professional and private life of Edyth von Haynau (alias Rosa Rosà) and the impact of her multidisciplinary artwork-drawings, paintings, ceramics, novels, and photographs-on art, society and politics in the $20^{\text {th }}$ century.

Two more contributions in this compilation are provided by researchers from our art history Max Planck Institutes. Philine Helas and Giorgia Gastaldon are both located at the Bibliotheca Hertziana in Rome. Giorgia Gastaldon looks at the discrimination of female artists in Italy in the 1960s. She focuses on the potential link between art made by women and the rise of feminist movements in Italy at that time. Gastaldon's research can be taken as a starting point for conducting broader research on the impact of gender stereotypes in the world of art.

Philine Helas also looks at gender roles in art-not as artists, but as subjects. Her research focuses on how different genders are represented in $14^{\text {th }}$ and $15^{\text {th }}$ century Italian art in the context of healthcare and taking care of the poor; topics that were frequently the subject of art during the Middle Ages. Based on the assumption that images and representational conventions tend to be transported and transformed over very long periods of time, Helas looks at the meaning behind, and impact of, the Max Planck Society's self-representation through the use of Minerva as its emblem.

Birgit Kolboske (Max Planck Institute for the History of Science) also refers to the culture of our research organization. Titled "Hierarchies", her contribution provides an overview of a research project on the history of women and gender equality in the Max Planck Society. The project high- 
lights two facts: first, there are very few women working as scientists, and second, the majority of women work in administrative roles and support male scientists.

Marina Chugunova (Max Planck Institute for Innovation and Competition) and her colleagues provide an overview of the latest empirical research on the gender gap in knowledge-intensive occupations from an economics perspective. Bringing together different scientific approaches with their own research, they conclude that it is imperative to fight the gender gap with well-tailored policies and to support gender equality at the institutional and individual level.

The article written by Elifcan Çelebi (Max Planck Institute for the Study of Societies) provides another fascinating look at gender aspects within the labor market. Celebi strengthens the link between policy studies and gender research by comparing gender related labor market policies in Turkey before and after the Justice and Development Party (AKP) came to power in 2002. She concludes that there has been a paradigm shift regarding labor market policies targeted at women.

Esra Sarioglu (Max Planck Institute for Human Development) also deals with gender equality in Turkey. Sarioglu puts the topic of vigilantism under the microscope in order to ask the bigger questions about the link between violence against women and sexism in Turkish society, where women face hostility from men.

Discrimination and violence against women is also the topic of Ju Young Lee from the Max Planck Institute for Biological Cybernetics. The author investigates the marginalization, sexualized objectification, stereotyping, and exclusion of women in science by analyzing cases of sexualized discrimination and harassment that took place at science high schools in South Korea. Ju Young Lee calls on the scientific community-not only in Korea, but worldwide - to undertake efforts to implement an inclusive culture in the scientific community.

Gunda Wössner (Max Planck Institute for the Study of Crime, Security and Law) provides an overview of research projects at the Institute's Department of Criminology which investigate issues related to dealing with sex offences. She focuses on a project which evaluates the treatment of convicted sex offenders, identifies risk factors for reoffending, and looks at the lives of sex offenders after their release from prison.

Luisa Stella de Oliveira Coutinho Silva (Max Planck Institute for European Legal History) is also a legal scholar. She scrutinizes the handling of the category "gender" in women's legal historiography. Using the historical writing of the Brazilian state of Paraíba as an example, she demonstrates that women's legal history in colonial Brazil cannot be understood 
without interlacing gender, religion, social position and status. Against this background, she develops a unique approach for women's colonial legal history.

It might seem that this collection of articles has been compiled in a rather random manner. On the contrary, it represents the wide range of research fields and topics that are to be found under the umbrella of the Max Planck Society. To illustrate both the immense diversity of the individual projects and their relation to the direct research environment, a brief presentation of each researcher's institute and the current research framework accompanies each of the contributions.

Furthermore, this anthology is not designed to be an exhaustive, or even representative compilation of Max Planck research projects that include a gender dimension. Rather, the aim of this publication is to encourage the continuation of an increasing attention for gender aspects in basic research and within the Max Planck Society.

Ulla Weber

\section{REFERENCES}

Bohnet, Iris (2016). What Works: Gender Equality by Design. Cambridge: Harvard University Press.

Criado-Perez, Caroline (2019). Invisible Women: Exposing Data Bias in a World Designed for Men. New York: Abrams Press. 